\title{
Integration ambulant- stationärer Versorgung zur Sicherstellung medizinischer Grundversorgung in der Fläche
}

\section{GERALD GAß}

Dr. Gerald Gaß ist

Geschäftsführer des

Landeskrankenhaus

(Anstalt öffentlichen Rechts)

mit Sitz in Andernach
Integrierte Versorgung scheint als Allheilmittel aller tatsächlichen oder vermuteten Versorgungsmängel im deutschen Gesundheitswesen zu taugen. Tatsächlich hat sich jedoch nicht wirklich viel verändert seit der Einführung der Disease Management Programme oder der IV-Verträge im Sozialgesetzbuch V. Die sektorübergreifende Zusammenarbeit in getrennten Systemen agierender Akteure im Interesse einer qualitativ besseren oder effizientern Versorgung der Patienten scheitert in der Regel an den unterschiedlichen Interessen und dem vorhandenen Misstrauen. Anders dagegen dort, wo die Ressourcen knapp sind und Kooperation die einzige Strategie zum Überleben darstellt.

\section{Einleitung}

Wenn Sie heute eine Umfrage unter den Experten der Gesundheitspolitik machen, wo der besondere Handlungsbedarf zur Weiterentwicklung der Versorgungsstrukturen besteht, werden Sie mit Sicherheit bei mindestens neun von zehn Antwortenden den Begriff der Integrierten Versorgung hören. Der Mangel an Integrierten Versorgungsformen scheint ein zentraler Aspekt zu sein, wenn es darum geht, Qualität und Effizienz der Gesundheitsversorgung zu verbessern. Nach zwei Jahrzehnten intensiver Analyse, zahlreichen Sachverständigengutachten und ungezählten Stunden poli- tischer Debatte rund um die Integrierte Versorgung muss offensichtlich etwas dran sein an dieser Problembeschreibung.

Sehr viel schwieriger stellt sich offensichtlich die konkrete Beschreibung dessen dar, was als Zielvorstellung vor dem inneren Auge erscheint, wenn von Integrierten Versorgungsstrukturen die Rede ist. Da ist zunächst einmal die Vision der sektorübergreifenden Versorgungskette, die idealer Weise von der Prävention über die ambulante und akutstationäre Versorgung bis hin zu Rehabilitation und Pflege den Versorgungsbedarf meist chronisch Kranker organisiert. 
Dort werden Informationen zwischen den Sektoren ausgetauscht, Doppeluntersuchungen vermieden und Mauern finanzieller Fehlanreize niedergerissen. Die Realität des deutschen Gesundheitswesens holt diese Vorstellungen, die eher mit einem einheitlichen nationalen $\mathrm{Ge}$ sundheitswesen korrespondieren als mit einem selbstverwalteten System, ziemlich schnell ein.

Unser System ist sehr viel komplexer organisiert, vielleicht $\mathrm{zu}$ komplex, um die oben skizzierte Vision einer Integrierten Versorgung chronisch Kranker mit Leben erfüllen zu können. Trotz vielfacher Reformanstrengungen des Gesetzgebers, die in den letzten zwanzig Jahren immer auch das Ziel der Integrierten Versorgung auf der Agenda hatten, sind wir nicht wirklich weiter gekommen. Noch immer setzen wir auf (getrennte) Selbstverwaltungsstrukturen, auf die duale Krankenhausfinanzierung, die Zuständigkeit der Länder für die Krankenhausplanung, die Verantwortung der Kassenärztlichen Vereinigungen für die Versorgungsplanung und Sicherstellung im ambulanten Sektor und auf die meist geteilte Kostenverantwortung bei Prävention, Akut-, Rehaversorgung und Pflege. Das in diesem, für den gesunden Menschenverstand, unüberschaubaren Gefecht von Zuständigkeit, Macht, Einfluss und Kostenverantwortung das gemeinsame Interesse für die sektorübergreifende Integrierte Versorgung von Patienten, wenn überhaupt nur auf dem kleinsten gemeinsamen Nenner erreicht wird, ist keine echte Überraschung. In den wenigen positiven Beispielen, in denen es wirklich gelungen ist, etwas auf die Beine zu stellen, was diesen Namen verdient und nachhaltig den Patienten mehr Qualität und Effizienz in der Behandlung ihrer Erkrankung bringt, ist dies nicht wegen, sondern trotz des bestehenden Systems gelungen. Abhängig ist dieser Erfolg im Einzelnen dann immer von handelnden Personen, die zumindest vorübergehend ein gemeinsames $\mathrm{Ziel}$ über den eigenen Vorteil oder den ihrer Organisation gestellt haben.

Eine spannende Ausnahme ganz anderer Systemvoraussetzungen für den Erfolg sektorübergreifender Integrierter Versorgungsstrukturen hält das deutsche System aber dann doch bereit, die Psychiatrie. Hier werden ganze Re- gionen mit mehreren hunderttausend Einwohnern durch ein sektorübergreifendes System von Institutsambulanzen, Tageskliniken, Tagesstätten, Akutversorgungsangeboten, ambulanter Pflege und Wohnangeboten eines einzigen Trägers versorgt. Die sonst im somatischen Bereich bestehende Konkurrenz zu den freiberuflichen Niedergelassenen spielt

\section{Die im somatischen Bereich bestehende Konkurrenz zu den freiberuflichen Niederge- lassenen spielt in der Psychia- trie praktisch keine Rolle.}

praktisch keine Rolle, weil der Versorgungsbedarf bei weitem größer ist als die vorhandenen Angebote. Hier können bei entsprechenden Budgetvereinbarungen mit den Krankenkassen genau die Versorgungsstrukturen entstehen, die den Befürwortern der Integrierten Versorgung vorschweben. Dort entwickeln sich Strukturen, die gegen den allgemeinen Trend zu einem Abbau von akutstationären Angeboten und einem Zuwachs teilstationärer und ambulanter wohnortnaher Strukturen führen. Wer dies genauer betrachten möchte, dem sei ein Blick in den Norden der Republik auf die Regionalbudgets der Psychiatrie in Schleswig-Holstein ans Herz gelegt.

Es ist sicher realistisch, davon auszugehen, dass die Grundstrukturen des deutschen Gesundheitswesens auch in den nächsten zwei Jahrzehnten ebenso wenig grundsätzlich in Frage gestellt werden, wie das in den letzten zwanzig Jahren der Fall war. Insoweit müssen wir uns damit abfinden, dass Integrierte Versorgung eine schwer verdauliche Mahlzeit bleiben wird, an der auch zukünftig Sachverständige, politisch Verantwortliche, Mandatsträger der Selbstverantwortung und Leistungserbringer zu kauen haben werden. $\mathrm{Ob}$ es dabei gelingt, den Patienten stärker als bisher in den vielzitierten „Mittelpunkt" zu stellen, bleibt abzuwarten.

\section{Sektorübergreifende Integration vor dem Hintergrund von regionaler Unterversorgung}

Immer dann, wenn der Markt für Gesundheitsleistungen von Überversorgung gekennzeichnet ist, wird es besonders schwierig mit dem Ziel der Integrierten Versorgung. Wenn sich mehrere Krankenhäuser und fachärztliche ambulante Praxen einer Region im Wettbewerb um eine begrenzte Zahl chronisch kranker Patienten gegenüber stehen, wird der Versuch der Kooperation stets auch vom Misstrauen des Wettbewerbs begleitet. Der Partner des einen ist schnell der Gegner des anderen. Andererseits kann die Integration von ambulanter und stationärer Versorgung in einer konkreten Region unter den gegebenen Rahmenbedingungen eine zwingende und praktisch alternativlose Form der Versorgung darstellen, wenn ein absoluter Mangel an Versorgungsangeboten existiert. Doch auch in einer solchen Unterversorgungssituation bedarf es einer weiteren Bedingung, um eine regionale Integration auf den Weg zu bringen, nämlich dem erklärten Ziel des Erhalts wohnortnaher Versorgungsstrukturen. Nur wenn es ein klares Bekenntnis aller Verantwortlichen zu diesem Ziel gibt, werden die regionalen Versorgungsanbieter sich dieser Integrationsherausforderung stellen. In der Situation der Unterversorgung bei gleichzeitig erklärtem Willen, Versorgungsstrukturen nicht gänzlich aufzugeben, entsteht eine Dynamik, die unter den gegebenen Bedingungen an der Integration von ambulanten und stationären Versorgungsstrukturen nicht vorbeikommt.

Unterversorgung kann in einem Umfeld, das von Berufs- und Niederlassungsfreiheit geprägt ist, nur durch Kreativität und Attraktivität beseitigt werden. Wenn man die knappe Ressource Facharzt in unterversorgte Gebiete bringen möchte, muss man ihn (oder heute meistens sie) locken. Doch wer glaubt, dies funktioniere mit Honorarzuschlägen der Kassenärztlichen Vereinigung oder günstigen Praxisräumen der Gemeinde, der täuscht sich gewaltig. Solche Verlockungen reichen bei weitem nicht aus, weil es längst nicht mehr nur um materielle Anreize geht, die die Fachärztin/den Facharzt in die Region bringt. Attraktivität ist mehr. Man braucht ein schlüssiges und glaubwürdig nachhaltiges Gesamtkonzept für die medizinische Versorgung in der Region. Wer schon einmal den Versuch gemacht hat, eine frei werdende Facharztpraxis am Rande einer Ballungsregion nachzubesetzen, 
weiß, wovon die Rede ist. Niemand, außer den immer seltener werdenden Idealisten, trifft eine auf Jahrzehnte angelegte Lebensentscheidung gegen den Trend der Zentralisierung in die Ballungsgebiete. Es sei denn, man bietet genau dieses überzeugende Konzept an.

Man muss sich nur die unzähligen Umfragen unter Medizinern und Medizinstudierenden anschauen, um zu er-

\section{Der Erhalt von kleineren Krankenhäusern im ländlichen Raum hat für die betroffene Region bedeutende Vorteile.}

und ist auch nur durch immensen Aufwand wieder zu beleben.

Dem aufmerksamen Beobachter dürfte nicht entgangen sein, dass sich zuletzt ein Umdenken in der öffentlichen Debatte um stationäre Versorgungsstrukturen abzeichnet. War es zu Beginn des DRG-Zeitalters noch schick, die kleinen Krankenhäuser in der Fläche zugunsten der Zentren mit hohen Fallzahlen tot zu schreiben, setzt sich zunehmend die Erkenntnis durch, dass Größe allein kein Markenzeichen für Effizienz, Qualität und bedarfsgerechte Versorgung ist. Auch die aktuell aufflammende Debatte um steigende Fallzahlen (in Zentren) und deren

kennen, wo die Bedingungen, aber auch die Chancen für ein sektorübergreifendes regionales Gesamtkonzept liegen. Mann/Frau wünscht sich: Arbeitszeiten, die Beruf und Familie vereinbaren lassen, einen fachlichen Austausch, ein vielfältiges Arbeitsgebiet, möglichst wenig Bürokratie und Verwaltungsaufwand. Vorstellungen, die sich nur schwer mit dem freiberuflichen Betrieb einer Einzelpraxis in unterversorgten Regionen in Einklang bringen lassen.

\section{Regionale Integration im Interesse der Bevölkerung}

Die Annahme vorausgesetzt, dass auch kleinere Krankenhäuser im ländlichen Raum in der Lage sind, im therapeutischen Spektrum der medizinischen Grundversorgung medizinische Leistungen mit hoher Qualität anzubieten, hat der Erhalt solcher Angebote bedeutende Vorteile für die betroffene Region. Die Bürgerinnen und Bürger haben, entgegen aller Unkenrufe, ein großes Vertrauen in „ihre“ regionalen Anbieter, in die niedergelassene Ärzteschaft ohnehin, aber auch in die kleinen Grundversorgungskrankenhäuser, solange keine hochkomplexen Therapien erforderlich sind, die, ohne Zweifel, in den dafür spezialisierten Zentren behandelt werden sollten. Wohnortnahe medizinische Grundversorgung ist nicht nur auf dem Papier der Regionalplanung ein Standortfaktor, sondern auch ganz konkret in den Erwartungen der Einwohner dieser Regionen. Wenn dieser Standortfaktor bewusst oder unbewusst durch Untätigkeit aufgegeben wird, geht viel verloren medizinische Notwendigkeit im Einzelfall lässt sich eher an der akutstationären Überversorgung in Ballungsgebieten festmachen, als an dem kleinen Grundversorgungskrankenhaus in der Fläche.

\section{Grundversorgungskliniken als Motor regionaler Integrationsstrukturen}

Bei genauerer Regionalanalyse zeigt sich ein interessanter Zusammenhang: In den Regionen, die die typischen Merkmale klassischer „Unterversorgungsregionen“ tragen (zurückgehende Bevölkerung, Alterung über dem allgemeinen Trend, dünnere Besiedlung, unterdurchschnittliche Verkehrsinfrastruktur, größere Entfernung zu Ballungsgebieten), ist die tatsächliche Ausstattung mit medizinischen Versorgungsangeboten dennoch relativ unterschiedlich. Wenn es in diesen Gebieten regionale Grundversorgungskrankenhäuser gibt, fungieren diese vielfach als Kern oder Magnet einer regionalen ambulanten Versorgungsstruktur. War dies in der Vergangenheit eine eher zufällige Funktion, die so vom Krankenhausträger nicht bewusst wahrgenommen oder gar herbeigeführt wurde, erleben wir heute vielfach eine sehr aktive Rolle der Krankenhäuser, die genau diese Aufgabe als ein explizites Unternehmensziel begreifen.

An diesen Krankenhausstandorten hängen meist weitere wichtige medizinische Versorgungsangebote, wie die regionale Notarztversorgung oder die ambulanten Bereitschaftsdienstzentralen zur Versorgung außerhalb der üblichen Sprechstundenzeiten. Grundver- sorgungskrankenhäuser in der Fläche verfügen über die Möglichkeiten, regionale Integrierte Versorgungsstrukturen zu befördern und als Motor mit zu entwickeln. Die Handlungsmöglichkeiten dazu hat der Gesetzgeber bewusst oder unbewusst deutlich gestärkt. Wesentlich zu nennen sind die gleichzeitige Beschäftigung von Ärzten im stationären und ambulanten Sektor, die Gründung von Filialpraxen, die Beschäftigung von Honorarärzten zum Betrieb von Hauptfachabteilungen und die Einführung von medizinischen Versorgungszentren. Ausdrücklich nicht zur Stärkung dieser Handlungsmöglichkeiten zählt interessanterweise die Einführung Integrierter Versorgungsverträge nach $\$ 140$ SGB V. Sie spielen in unterversorgten Regionen praktisch keine Rolle.

\section{Ambulant-stationäre Versorgung in der Region - ein Praxisbeispiel}

Den Versuch, in einem komplexen Gesamtkonzept alle Formen der sektorübergreifenden Zusammenarbeit in einer „Unterversorgungsregion“ $\mathrm{zu}$ verwirklichen, übernimmt aktuell das Landeskrankenhaus (Anstalt öffentlichen Rechts) als öffentlicher Krankenhausträger in Rheinland-Pfalz an seinem Standort in Meisenheim am Glan. In diesem nachfolgend $\mathrm{zu}$ beschreibenden Gesamtkonzept ist das Ziel der Stabilisierung und Wiederbelebung der ambulanten fachärztlichen Versorgungsstrukturen ausdrücklich formuliert und konstitutiver Bestandteil des Konzepts. In der gemeinsamen Betrachtung aller am Vorhaben Beteiligten wird die medizinische Versorgung der Region zukünftig nur dann nachhaltig gesichert werden können, wenn die bisher weitgehend getrennten ambulanten und akutstationären Strukturen funktional, personell und organisatorisch zusammengeführt werden.

Seit dem Jahr 2003 ist das Landeskrankenhaus (AöR) Träger eines kleinen Grundversorgungskrankenhauses in der Stadt Meisenheim am Glan (3.000 Einwohner). Es handelt sich um ein 70 Betten Haus, das vom ehemaligen konfessionellen Träger in einer Phase übernommen wurde, in der mangels Zukunftsperspektive die Schließung drohte. Die im weiteren regionalen Umfeld nächsten Krankenhausstandorte liegen zwischen 30 und 45 Minuten Fahrzeit entfernt. 
Eine Autobahn- oder Bahnverbindung existiert nicht. Das ausschließlich aus Belegabteilungen (Chirurgie, Innere Medizin, Gynäkologie/Geburtshilfe, Anästhesie) bestehende Haus wurde schon damals in der Zusammenarbeit mit freiberuflich niedergelassenen Medizinern als Belegärzten betrieben. Unmittelbar nach dem Trägerwechsel stellte sich durch den Weggang des Gynäkologen die Nachfolgefrage, die schon 2003 nicht beantwortet werden konnte. Trotz intensiver Suche konnte kein Belegarzt für diese Abteilung gefunden werden. Auch aufgrund der schon damals niedrigen Geburtenzahlen lag die Schließung dieser Abteilung letztlich nahe und war am Ende unvermeidlich.

Am gleichen Standort, aber räumlich etwa zwei Kilometer entfernt, verfügte das Landeskrankenhaus (AöR) zum Zeitpunkt der Übernahme über zwei weitere Einrichtungen, eine neurologischen Fachklinik mit Akut- und Rehaversorgung (80 Betten) und ein überregional tätiges Sprachheilzentrum zur stationären Therapie für Kinder und Jugendliche.

In einem ersten Schritt der Integration des neuen Krankenhausstandortes in die Trägerstruktur entstand die Glantal-Klinik Meisenheim mit zwei Betriebsstätten, aber einer gemeinsamen Führungs- und Organisationsstruktur. Darüber hinausgehende inhaltliche Veränderungen der Versorgungsschwerpunkte gab es zunächst nicht. Im Jahr 2008 wurde durch die Geschäftsführung des Träger die Zukunftsentwicklung der Klinik, auch bedingt durch zunehmende wirtschaftliche Schwierigkeiten am Standort, mit hoher Priorität auf den Plan gerufen. Es mussten dringende Entscheidungen zu einer baulichen Sanierung der beiden Betriebsstätten und idealer Weise der Zusammenführung an einem gemeinsamen Standort getroffen werden. Dem Träger war bewusst, dass derart umfassende Investitionsentscheidungen nur vor dem Hintergrund eines tragfähigen inhaltlichen Zukunftskonzeptes fallen konnten.

Eine Arbeitsgruppe aus Standortverantwortlichen und Trägervertretern entwickelte über Monate hinweg in einem intensiven Prozess verschiedene Alternativen, aus denen dann ein bevorzugtes Zukunftsszenario extrahiert wurde. Mit diesem Konzept, das inhaltlich beschrieben und wirtschaftlich kalkuliert war, wurden dann in einem weiteren Schritt Gespräche mit dem Ministerium, als zuständige Krankenhausplanungsbehörde, den Krankenkassen, als Kostenträger, und der Kassenärztlichen Vereinigung, als verantwortlichem Akteur im ambulanten Sektor, geführt. Parallel dazu wurde bei den Kommunalpolitikern und örtlichen Landtagsabgeordneten für das Zukunftskonzept eines sektorübergreifenden Gesundheitszentrums geworben. In Informationsschreiben und Gesprächen gelang es, die niedergelassenen Ärzte in der Region für das Vorhaben zu interessieren. Das schließlich definierte und in der Umsetzung befindliche $\mathrm{Zu}$ kunftskonzept setzt in vollem Umfang auf die Integration ambulanter und stationärer Leistungen in einem „Gesundheitszentrum“ und ist, wie es heute so oft in der Politik heißt, im Hinblick auf seinen integrativen Ansatz, alternativlos. Das Gesamtkonzept schöpft die heutigen gesetzlichen Möglichkeiten der gemeinsamen und sektorübergreifenden Leistungserbringung voll aus und geht dank einer innovativen Landeskrankenhausplanung auch noch darüber hinaus.

Konkret setzt das Versorgungskonzept am regionalen Bedarf der örtlichen Bevölkerung an und respektiert die besondere Kompetenz überregionaler Schwerpunkt- und Maximalversorgungsanbieter. Die akutstationäre Versorgung konzentriert sich deshalb auf die bereits bestehenden chirurgischen und internistischen Belegabteilungen, ergänzt um das seit Jahrzehnten etablierte Angebot der Neurologie im Rah- men einer Hauptfachabteilung. Komplettiert wird das Versorgungsangebot schon heute durch den Notarztstandort und die Bereitschaftspraxis der Kassenärztlichen Vereinigung am Krankenhaus. Neu ist die geplante Etablierung einer interdisziplinären Belegabteilung, die wohnortnah akutstationäre Therapien aus den Disziplinen HNO, Urologie, Gynäkologie und Orthopädie anbieten wird. Diese interdisziplinäre Abteilung wird, wie die beiden Abteilungen Chirurgie und Innere Medizin, in der Zusammenarbeit mit niedergelassenen oder im ambulanten Sektor angestellten Medizinern betrieben. Der Krankenhausträger favorisiert dabei die Zusammenarbeit mit Filialpraxen, die sich im ambulanten Teil des Gesundheitszentrums ansiedeln sollen und die dortige Infrastruktur bedarfsgerecht in Teilzeit nutzen und so wirtschaftlich auslasten. Notwendige Bereitschaftsdienste über alle Abteilungen hinweg werden kooperativ und interdisziplinär geleistet. Der Facharztstandard wird über die Rufbereitschaften mit den ambulanten Medizinern gewährleistet.

Die fachärztlichen Filial- oder Zweigpraxen, die im Rahmen einer Reform der damaligen Gesundheitsministerin, Ulla Schmidt, ins Gesetz aufgenommen wurden, bieten eine große Chance zur fachärztlichen Versorgung in der Fläche. Realistisch ist deren Ansiedlung allerdings nur, wenn sie nicht singulär, sondern in Kooperation mit weiteren Praxen oder einem Krankenhaus betrieben werden. Nur dann können sie

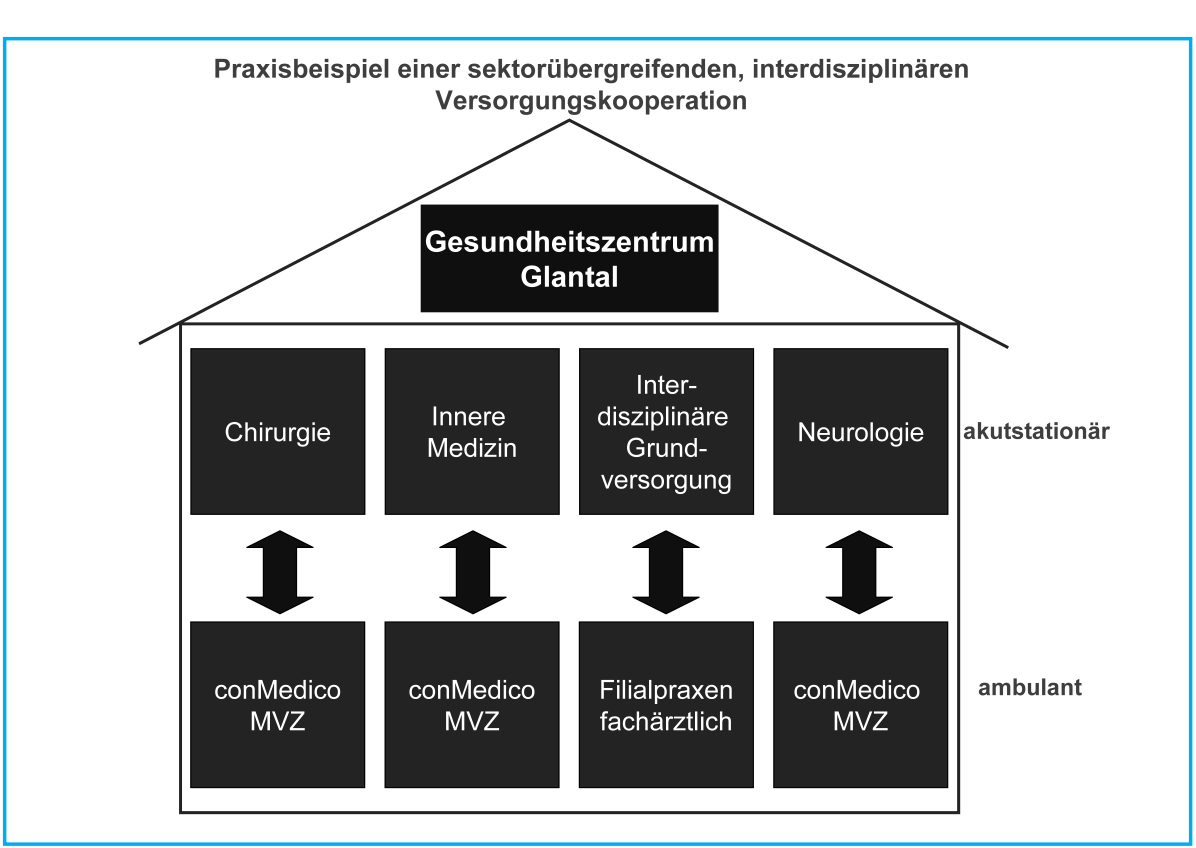


von der gemeinsamen Infrastruktur und Logistik im diagnostischen und therapeutischen Bereich profitieren.

Die ehemals freiberuflichen Arztsitze, die in der Vergangenheit den Betrieb der bestehenden Belegabteilungen (Chirurgie, Innere Medizin) ermöglichten, wurden bereits in den zurückliegenden beiden Jahren schrittweise in ein Medizinisches Versorgungszentrum des Krankenhausträgers integriert. Unmittelbarer Anlass für diese Umstellung waren die unübersehbaren Nachbesetzungsschwierigkeiten dieser freiwerdenden Praxen, verbunden mit dem Risiko der Verlagerung ins nächste Mittelzentrum. Diese neue Form der ambulanten Leistungserbringung im MVZ ist attraktiv für Mediziner, die den unternehmerischen Part einer Niederlassung scheuen. So konnten angestellte Ärzte gefunden werden, mit denen nun neben der ambulanten Versorgung auch der Betrieb der beiden Belegabteilungen sichergestellt werden kann. Das in der Vergangenheit immer bestehende Risiko für den Krankenhausträger, die freiberuflichen Beleger zu verlieren und damit die akutstationäre Versorgung zumindest vorübergehend einstellen zu müssen, ist damit deutlich reduziert.

Das Konstrukt des Medizinischen Versorgungszentrums hat nicht nur in diesem Fall Vorteile gegenüber der freiberuflichen Praxis; auch in vielen wei-

\section{Zur regionalen Integration der Versorgung braucht es ein auf die konkreten Bedarfe und Möglichkeiten einer Region abgestimmtes Versorgungskonzeptes.}

teren Konstellationen ist es in Sachen Attraktivität einer freiberuflichen Niederlassung überlegen und kann damit ärztliche Ressourcen bereithalten, die ansonsten nicht zur Verfügung stehen. Dieses Modell einer regionalen interdisziplinären und sektorübergreifenden Kooperation zur Leistungserbringung ist nur denkbar unter bestimmten Voraussetzungen:

- Zunächst einmal braucht es einen Krankenhausträger, der die zentrale Rolle der Steuerung und Koordinati- on übernimmt. Er leistet damit logistische und investive Vorarbeit, ohne die die Kooperation der niedergelassenen Mediziner nicht möglich wäre.

- Der Existenz eines Medizinischen Versorgungszentrums, mit dem fachärztlicher Sachverstand abgedeckt werden kann, der über freiberufliche Niedergelassene nicht zur Verfügung steht.

- Eine Landeskrankenhausplanung, die den Erhalt der wohnortnahen Akutversorgung im Sinne einer bedarfsgerechten Grundversorgung als erklärtes Ziel aufnimmt und umsetzt. - Innovative regionale Partner, die sich einen gegenseitigen Vertrauensvorschuss auf dem Weg der Zielerreichung gewähren.

- Die finanzielle Attraktivität belegärztlicher Leistungserbringung.

- Die Flexibilität in der interdisziplinären Zusammenarbeit der Mediziner.

\section{Fazit}

An der sektorübergreifenden Leistungserbringung in tendenziell unterversorgten Regionen führt kein Weg vorbei. Knappheit zwingt zu Kreativität. Der Ärztemangel, der vor allem und zunächst in den ländlichen Regionen spürbar ist, zwingt dazu, dieses „knappe Gut“ intersektoral einzusetzen. Wer das Ziel verfolgt, in dünner besiedelten ländlichen Regionen wohnortnah zu versorgen, muss dem quantitativ geringeren Versorgungsbedarf auch ein entsprechendes Angebot entgegensetzen, das aber dennoch wirtschaftlich darstellbar und qualitativ wettbewerbsfähig ist.

Zur regionalen Integration der Versorgung braucht es weniger neue Planungsgremien der Selbstverwaltung oder der Politik, als vielmehr ein auf die konkreten Bedarfe und Möglichkeiten einer Region abgestimmtes Versorgungskonzeptes. Entscheidend für den Erfolg oder Misserfolg sind die Akteure in der Region. Es bedarf der Koordinierung und Steuerung eines solchen Vorhabens und des Vertrauens in eine faire und für alle Beteiligten vorteilhafte Zusammenarbeit.

Die Organisationsform, in der die sektorübergreifende Zusammenarbeit gelebt wird, ist nicht entscheidend. Die
Anzahl der unabhängigen und in eigener Rechtsform konstituierten Akteure erhöht jedoch die Komplexität des Vorhabens dramatisch. Neue rechtliche Rahmenbedingungen sind nicht zwingend erforderlich. Die bestehenden sollten aber nicht weiter eingeschränkt oder im Hinblick auf ihre finanzielle Attraktivität beschnitten werden.

Im September 2012 findet nach zweijähriger Bauplanung in Meisenheim am Glan der Erste Spatenstich zum Neubau des 40 Millionen Euro Projektes „Gesundheitszentrum Glantal“" statt. Gegen den Trend der Zentralisierung entsteht eine Versorgungseinrichtung, die durch ihren integrativen Charakter das Vertrauen der Investoren und Vertragspartner gewonnen hat. 